\title{
Diffractive production of quark-antiquark pairs
}

\section{Antoni SZCZUREK ${ }^{* \dagger}$}

University of Rzeszów, PL-35-959 Rzeszów, Poland, and

Institute of Nuclear Physics PAN, PL-31-342 Cracow, Poland

E-mail: Antoni.Szczurek@ifj.edu.pl

\section{Marta ŁUSZCZAK}

University of Rzeszów, PL-35-959 Rzeszów, Poland

E-mail: luszczak@univ.rzeszow.pl

\section{Wolfgang SCHÄFER}

Institute of Nuclear Physics PAN, PL-31-342 Cracow, Poland

E-mail: Wolfgang.Schaferdifj.edu.pl

We discuss diffractive dissociation of gluons into heavy quark pairs. The particular mechanism is similar to the diffractive dissociation of virtual photons into quarks. The amplitude for the $g p \rightarrow Q \bar{Q} p$ is derived in the impact parameter and momentum space. The cross section for single diffractive $p p \rightarrow Q \bar{Q} p X$ is calculated as a convolution of the elementary cross section and gluon distribution in the proton. Integrated cross section and differential distributions in transverse momentum and rapidity of the charm and bottom quark and antiquark, etc. are calculated for the nominal LHC energy for different unintegrated gluon distributions from the literature. The ratio of the bottom-to-charm cross sections are shown and discussed as a function of several kinematical variables.

XXI International Workshop on Deep-Inelastic Scattering and Related Subject-DIS2013,

\footnotetext{
* Speaker.

$\dagger$ This work was supported by the Polish National Science Centre (on the basis of decision No. DEC2011/01/B/ST2/04535).
} 


\section{Introduction}

Hard diffractive production is characterized by the production of massive objects $\left(W^{ \pm}, Z^{0}\right.$, Higgs boson, pairs of heavy quark - heavy antiquark) or objects with large transverse momenta (jets, dijets) and one (single diffractive process) or two (central diffractive process) rapidity gaps between proton(s) and the centrally produced massive system. The cross section for these processes is often calculated in terms of hard matrix elements for a given process and so-called diffractive parton distributions. The latter are often calculated, following a suggestion of Ingelman and Schlein[1], in a purely phenomenological approach in terms of parton distributions in the pomeron and a Reggetheory motivated flux of Pomerons.

Diffractive production of heavy quarks was previously discussed within the Ingelman-Schlein model in Refs. $[2,3,4,5]$ and proposed as a probe of the hard substructure of the Pomeron.

In this presentation we discuss a specific mechanism for the diffractive production of heavy quark - antiquark pairs in proton-proton collisions in a "microscopic approach" which does not use the assumptions of Regge factorization, and in which the QCD Pomeron is rather modelled by exchange of a gluon ladder related to the unintegrated gluon distribution in the proton.

The mechanism we propose is based on the partonic subprocess $g p \rightarrow Q \bar{Q} p$ - the diffractive dissociation of a gluon into a heavy quark pair. The forward amplitude for the $g p \rightarrow Q \bar{Q} p$ is well behaved and is perturbatively calculable without introducing new soft parameters.

In the usual treatment of hard diffraction, heavy quarks are generated from gluons in the Pomeron and a valence-like heavy quark contribution is not present. In this sense the mechanism discussed here is complementary to existing approaches, although eventually we intend that the Ingelman-Schlein mechanism be superseded also by a microscopic model for the gluon distribution in the Pomeron.

The generic mechanism of the reaction is shown in Fig.1. In this approach $Q \bar{Q}$ pairs are produced in the Pomeron fragmentation region, close to the rapidity gap, whereas gluon-fusion populate a large part of the phase space taken up by the diffractively produced system and will generally give a tiny contribution in the Pomeron fragmentation region, unless there are a lot of hard gluons in the Pomeron.
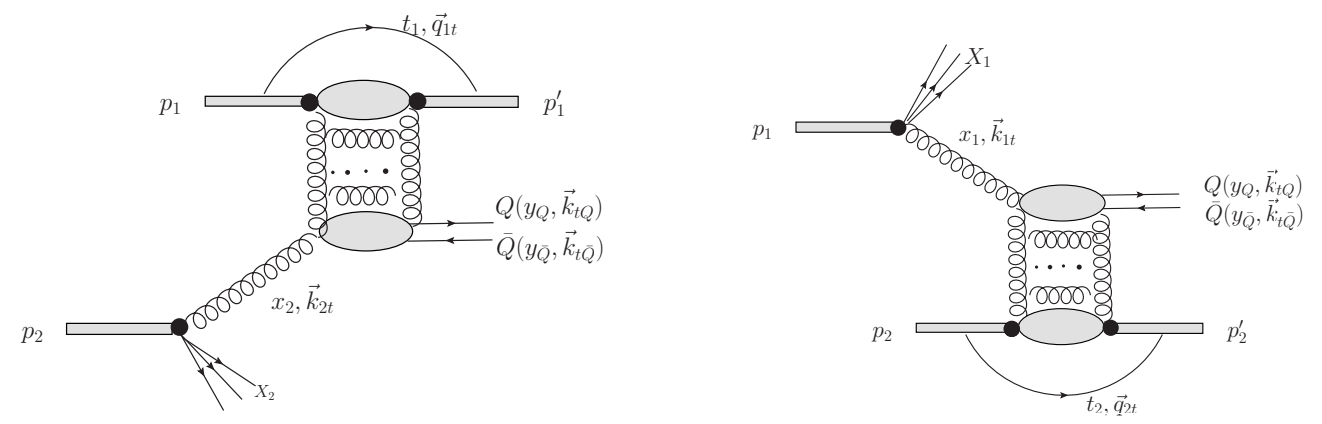

Figure 1: The mechanism of gluon dissociation into $Q \bar{Q}$ via exchange of gluonic ladder in proton-proton collisions.

The mechanism discussed here was considered previously in $[6,7]$ in an approximation in which gluon transverse momenta in the Pomeron are integrated out. Our results appear to be 
different from those presented in [6, 7]. Somewhat related, but different, microscopic mechanisms were discussed in [8]).

In our recent paper [9] we have presented the amplitude for the $g p \rightarrow Q \bar{Q} p$ (sub)process and calculateed integrated and differential cross section for the $p p \rightarrow Q \bar{Q} p X$ single-diffractive processes at the LHC. Here we show only some selected results.

\section{Sketch of the formalism}

It is shown in our recent paper [9] how to obtain the amplitude for the $g p \rightarrow Q \bar{Q} p$ process. The differential parton-level cross section can be written as:

$$
\left.16 \pi \frac{d \hat{\sigma}(g N \rightarrow Q \bar{Q} N ; \hat{s})}{d \Delta^{2}}\right|_{\Delta^{2}=0}=\frac{1}{2 \cdot\left(N_{c}^{2}-1\right)} \cdot \sum_{\lambda_{g}, \lambda, \bar{\lambda}, a}\left|\mathscr{A}_{D}\left(g_{\lambda_{g}}^{a} N \rightarrow Q_{\lambda} \bar{Q}_{\bar{\lambda}} N\right)\right|^{2} d z \frac{d^{2} k}{(2 \pi)^{2}}
$$

and the final multi-dimensional cross section reads:

$$
\left.\frac{d \hat{\sigma}(g N \rightarrow Q \bar{Q} N ; \hat{s})}{d z d^{2} k d \Delta^{2}}\right|_{\Delta^{2}=0}=\frac{\pi}{4 N_{c}^{2}\left(N_{c}^{2}-1\right)^{2}} \alpha_{S}\left\{\left[z^{2}+(1-z)^{2}\right] \Phi_{1}^{2}+m_{Q}^{2} \Phi_{0}^{2}\right\} .
$$

The auxiliary functions $\Phi_{0}$ and $\Phi_{1}$ are defined in Ref.[9]. and Finally we calculate the spectrum of quarks in the $p p$-collision. Starting from the diffractive $g p \rightarrow Q \bar{Q} p$ cross section

$$
\left.\frac{d \hat{\sigma}(g N \rightarrow Q \bar{Q} N ; \hat{s})}{d z d^{2} k d \Delta^{2}}\right|_{\Delta^{2}=0}=\hat{f}_{Q \bar{Q}}(z, k ; \hat{s})
$$

we can obtain the corresponding cross section for $p p$-collisions in the collinear approximation for the incoming gluon as:

$$
\begin{aligned}
\left.\frac{d \sigma(p p \rightarrow X Q \bar{Q}+p ; s)}{d x_{Q} d^{2} k d \Delta^{2}}\right|_{\Delta^{2}=0} & =\int d x d z \delta\left(x_{Q}-x z\right) g\left(x, \bar{Q}^{2}\right) \hat{f}_{Q \bar{Q}}(z, k ; x s) \\
& =\int_{x_{Q}}^{1} \frac{d x}{x} g\left(x, \bar{Q}^{2}\right) \hat{f}_{Q \bar{Q}}\left(\frac{x_{Q}}{x}, k ; x s\right)
\end{aligned}
$$

We can also calculate the fully differential distribution in $x_{Q}, x_{\bar{Q}}=x-x_{Q}$, rapidities etc.

$$
\left.\frac{d \sigma(p p \rightarrow X Q \bar{Q}+p ; s)}{d x_{Q} d x_{\bar{Q}} d^{2} k d \Delta^{2}}\right|_{\Delta^{2}=0}=\frac{1}{x_{Q}+x_{\bar{Q}}} g\left(x_{Q}+x_{\bar{Q}}, \bar{Q}^{2}\right) \hat{f}_{Q \bar{Q}}\left(\frac{x_{Q}}{x_{Q}+x_{\bar{Q}}}, k ; x s\right) .
$$

\section{Results}

In our numerical calculations, we shall use three different UGDFs from the literature. One of them from Ref.[10] (labelled Ivanov-Nikolaev) is a fit to HERA structure functions data. The other two, from Ref.[11] (labelled Kutak-Stasto) are obtained by solving a BFKL equation accounting for subleading terms. One of the latter UGDFs also accounts for a nonlinear Balitsky-Kovchegov type term in the evolution equation. Both UGDF sets give a reasonable description of deep inelastic structure functions at small $x$. 

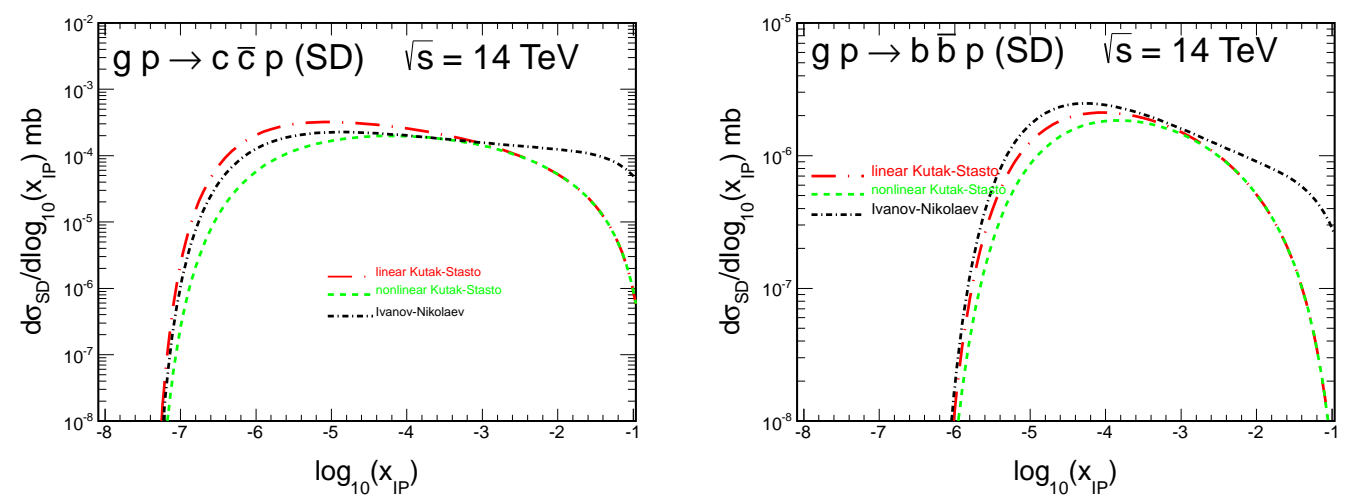

Figure 2: Distribution in $\log _{10}\left(x_{\mathbb{P P}}\right)$ for $c \bar{c}$ (left) and $b \bar{b}$ (right) produced in a single diffractive process for center of mass energy $\sqrt{s}=14 \mathrm{TeV}$ for the Ivanov-Nikolaev (solid), linear Kutak-Staśto (dashed) and nonlinear Kutak-Staśto (dotted) UGDFs. Absorptive effects have been included by multiplying by gap survival factor.

For the argument of running coupling constant we take: $\mu_{r}^{2}=M_{Q \bar{Q}}^{2}$ for $g \rightarrow Q \bar{Q}$ splitting and $\mu^{2}=\max \left(\kappa^{2}, k^{2}+m_{Q}^{2}\right)$ for the $t$-channel coupling of gluons to heavy quarks. For the quark masses, we take $m_{c}=1.5 \mathrm{GeV}$ and $m_{b}=4.75 \mathrm{GeV}$

Now differential distributions will be discused. Here, absorption corrections are included in a rough manner, by multiplying the cross section by a gap survival factor $S_{G}=0.05[12,13]$. A more subtle treatment, which would include the dependence of absorption effects on kinematical variables must be developed in the future.

Let us start with distributions in $x_{\mathbf{I P}}$ - the fractional longitudinal momentum loss of proton. Notice that $\log \left(1 / x_{\mathbf{I P}}\right)$ is proportional to the size of the rapidity gap. The cross section drops sharply at $x_{\mathbf{I P}} \lesssim 10^{-7}$ for charm quarks and $x_{\mathbf{I P}} \lesssim 10^{-6}$ for bottom quarks. This is related to the fact that with increasing gap size we are asking for harder partons in the dissociating proton. The gluon distribution however drops sharply at large $x$.

In the Ingelman-Schlein model, the gap size-dependence is described in terms of a universal flux of Pomerons. In our microscopic model the $x_{\mathbb{P}}$-dependence is driven by the dependence of the unintegrated gluon distribution on $x_{\text {eff }}=x_{\mathbf{I P}}$.

In Fig. 3 we present the rapidity distribution of charm (left panel) and bottom (right panel) quarks/antiquarks from diagram (b) in Fig.1. At large rapidities the cross section with the IvanovNikolaev UGDF is much larger than that with the nonlinear Kutak-Staśto UGDF. This is partially due to nonlinear effects included in the latter distributions. The nonlinear effects appear at $x_{\mathbf{I P}}<$ $10^{-4}$.

In Fig.4 we show transverse momentum distributions of charm (left panel) and bottom (right panel) quarks/antiquarks from one single-diffractive mechanism. The spread in transverse momentum here is somewhat smaller than in the Ingelman-Schlein model calculations of Ref.[5].

Now we wish to study dependence of the ratio of cross sections for $b \bar{b}$ and $c \bar{c}$ production as a function of some kinematical variables. Such ratios, to a good approximation, should be independent of absorption effects.

In Fig.5 we show the ratio as a function of quark rapidity. The ratio for the Ingelman-Schlein 

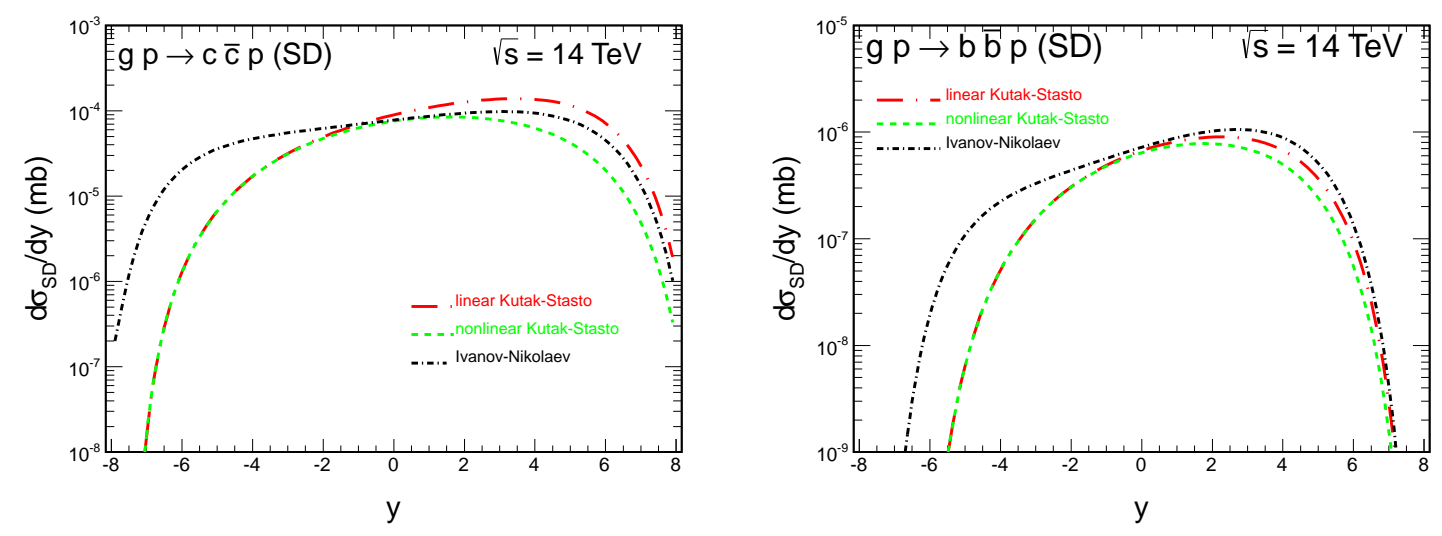

Figure 3: Distribution in rapidity of $c(\bar{c})$ (left) and $b(\bar{b})$ (right) produced in a single diffractive process for center of mass energy $\sqrt{s}=14 \mathrm{TeV}$. Absorptive effects have been included by multiplying by gap survival factor.
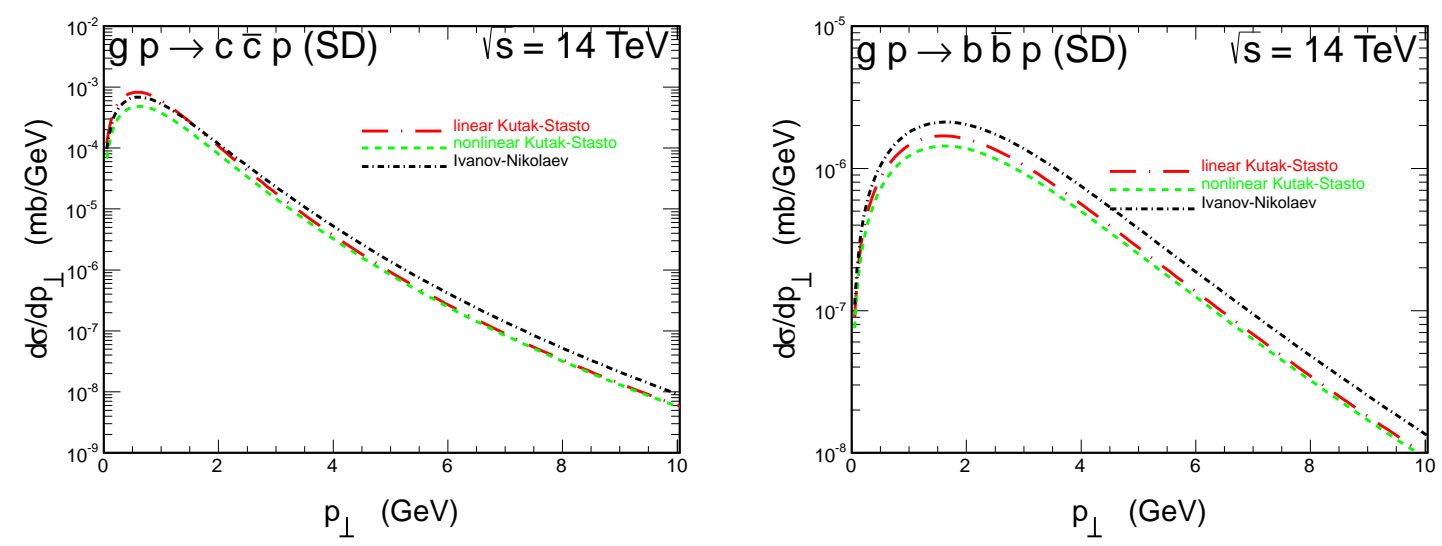

Figure 4: Distribution in transverse momentum of $c(\bar{c})$ (left) and $b(\bar{b})$ (right) produced in a single diffractive process for center-of-mass energy $\sqrt{s}=14 \mathrm{TeV}$. Absorptive effects have been included by multiplying by gap survival factor.

model is somewhat larger than that for the gluon-dissociation approach.

The charm-to-bottom ratio as a function of transverse momentum of the (anti)quark is shown in Fig.6. The ratio increases as a function of quark transverse momentum. The character of the function is in principle similar for both approaches.

\section{Conclusions}

Recently we have derived forward amplitudes for the $g p \rightarrow Q \bar{Q} p$ subprocess both in the impact parameter and momentum space representation in the forward scattering approximation. The amplitude for the off-forward directions within the diffraction cone was extrapolated by assuming exponential dependence known from other diffractive processes. The forward amplitude for the 


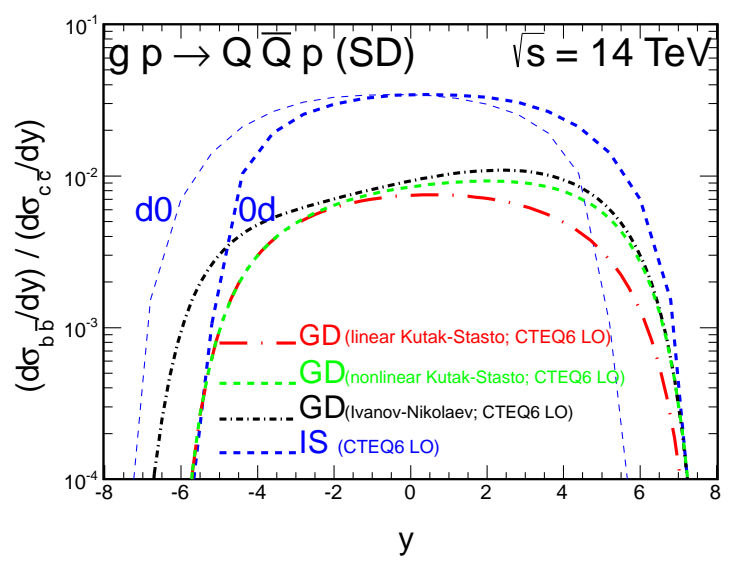

Figure 5: The ratio of the $b \bar{b}$ to $c \bar{c}$ distributions in quark (antiquark) rapidity.

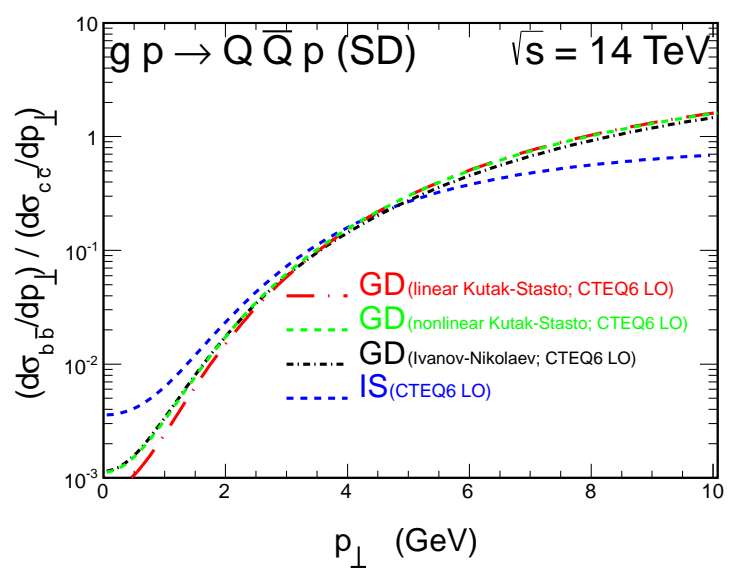

Figure 6: The ratio of the $b \bar{b}$ to $c \bar{c}$ distributions in quark (antiquark) transverse momentum.

$g p \rightarrow Q \bar{Q} p$ subprocess has been obtained in terms of unintegrated gluon distribution of the target proton.

The formulae have been used to calculate cross section for the single scattering process $p p \rightarrow$ $Q \bar{Q} p X$ as a convolution of the collinear gluon distributions in the proton and the elementary $g p \rightarrow$ $Q \bar{Q} p$ cross section both for charm and bottom production. When applied to the hadronic collisions, this approach allows one to predict heavy quark production "close to the rapidity gap".

We have presented here some results for the rapidity and transverse momentum distribution of quarks (antiquarks) at the nominal LHC energy $\sqrt{s}=14 \mathrm{TeV}$. The cross section for charm quarks is two orders of magnitude larger than that for bottom quarks, as expected from the $m_{Q}^{-4}$ scaling of the partonic subprocess.

We have calculated also ratio of the cross section for $b \bar{b}$ and $c \bar{c}$ as a function of several kinematical variables. The ratio is fairly smooth in (anti)quark rapidity and strongly depends on (anti)quark 
transverse momentum.

A measurement of the single diffractive production would be possible e.g. at ATLAS detector by using so-called ALFA detectors for measuring forward protons and their fractional energy loss and the main central detector for the measurement of $D$ or $B$ mesons. CMS+TOTEM is another option.

\section{References}

[1] G. Ingelman and P. E. Schlein, Phys. Lett. B 152, 256 (1985).

[2] H. Fritzsch and K. H. Streng, Phys. Lett. B 164, 391 (1985).

[3] M. Heyssler, Z. Phys. C73 299 (1997).

[4] M.B. Gay Ducati, M.M. Machado and M.V.T Machado, Phys. Rev. D81 054034 (2010).

[5] M. Łuszczak, R. Maciuła and A. Szczurek, Phys. Rev. D84 114018 (2011).

[6] G. Alves, E. Levin and A. Santoro, Phys. Rev. D 55, 2683 (1997), [hep-ph/9608443].

[7] F. Yuan and K. -T. Chao, Phys. Rev. D 60, 094012 (1999) [hep-ph/9810340].

[8] B. Z. Kopeliovich, I. K. Potashnikova, I. Schmidt and A. V. Tarasov, Phys. Rev. D 76, 034019 (2007) arXiv:0702106 [hep-ph].

[9] M. Łuszczak, W. Schäfer and A. Szczurek, arXiv:1305.4727[hep-ph].

[10] I. Ivanov and N.N. Nikolaev, Phys. Rev. D65 (2002) 054004.

[11] K. Kutak and A. Staśto, Eur. Phys. J. C41 (2005) 343.

[12] V. A. Khoze, A. D. Martin and M. G. Ryskin, Eur. Phys. J. C 18167 (2000), arXiv:0007359[hep-ph].

[13] U. Maor, AIP Conf. Proc. 1105248 (2009). arXiv:0811.2636 [hep-ph]. 